\title{
Standing up in multiple sclerosis (SUMS): protocol for a multi-centre randomised controlled trial evaluating the clinical and cost effectiveness of a home-based self-management standing frame programme in people with progressive multiple sclerosis
}

\author{
J. A. Freeman ${ }^{1 *}$, W. Hendrie ${ }^{1,2}$, S. Creanor ${ }^{3}$, L. Jarrett ${ }^{1,4}$, A. Barton ${ }^{5}$, C. Green ${ }^{6}$, J. Marsden ${ }^{1}$, E. Rogers ${ }^{1}$ and J. Zajicek ${ }^{7}$
}

\begin{abstract}
Background: Multiple sclerosis (MS) is an incurable, unpredictable but typically progressive neurological condition. It is the most common cause of neurological disability in young adults. Within 15 years of diagnosis, approximately $50 \%$ of affected people are unable to walk unaided, and over time an estimated $25 \%$ depend on a wheelchair. Typically, people with such limited mobility are excluded from clinical trials. Severely impaired people with MS spend much of their day sitting, often with limited ability to change position. In response, secondary complications can occur including: muscle wasting, pain, reduced skin integrity, spasms, limb stiffness, constipation, and associated psychosocial problems such as depression and lowered self-esteem. Effective self-management strategies, which can be implemented relatively easily and cheaply within people's homes, are needed to improve or maintain mobility and reduce sedentary behaviour. However this is challenging, particularly in the latter stages of disease. Regular supported standing using standing frames is one potential option.

Methods/Design: SUMS is a pragmatic multi-centre randomised controlled trial evaluating use of Oswestry standing frames with blinded outcome assessment and full economic evaluation. Participants will be randomly allocated (1:1) to either a home-based, self-management standing programme (with advice and support) along with their usual care or to usual care alone. Those in the intervention group will be asked to stand for a minimum of 30 min three times weekly over 20 weeks. Each participant will be followed-up at 20 and 36 weeks post baseline. The primary clinical outcome is motor function, assessed using the Amended Motor Club Assessment. The primary economic endpoint is quality-adjusted life years. The secondary outcomes include measures of explanatory physical impairments, key clinical outcomes, and health-related quality of life. An embedded qualitative component will explore participant's and carer's experiences of the standing programme.

(Continued on next page)
\end{abstract}

\footnotetext{
* Correspondence: jenny.freeman@plymouth.ac.uk

${ }^{1}$ Faculty of Health and Human Sciences, School of Health Professions,

Plymouth University, Peninsula Allied Health Centre, Derriford Rd, Plymouth PL6 8BH, England

Full list of author information is available at the end of the article
} 
(Continued from previous page)

Discussion: This is the first large scale multi-centre trial to assess the clinical and cost effectiveness of a home based standing frame programme for people who are severely impaired by MS. If demonstrated to be effective and cost-effective, we will use this evidence to develop recommendations for a health service delivery model which could be implemented across the United Kingdom.

Trial registration: ISRCTN69614598

Date of registration: 3.2.16 (retrospectively registered)

Keywords: Progressive Multiple Sclerosis, Standing, Standing frame, Physiotherapy, Self-management, Cost effectiveness, Mobility

\section{Background}

Multiple sclerosis (MS) is an incurable, unpredictable but typically progressive, life-long, neurological condition, affecting approximately 100,000 people in the United Kingdom (UK) [1]. It is the most common cause of neurological disability in young adults, with an estimated cost of $£ 1.4$ billion/annum to the National Health Service (NHS) and society [2]. Although most people start with a relapsing-remitting disease course, approximately two-thirds move to a progressive phase within eight years, at which point medical interventions are limited. Within 15 years of diagnosis, an estimated $50 \%$ of affected people are unable to walk unaided, and over time $25 \%$ become dependent on a wheelchair [3]. It is, therefore, unsurprising that mobility is a major concern for people with MS and health professionals. Surveys of people with MS consistently rank mobility as their highest priority $[4,5]$ and most important yet most challenging daily function [6]. Mobility has been correlated negatively with employment status and quality of life [2]. Evidence shows that enhancing physical activity and reducing sedentary behaviour can improve mobility and directly associated complications [7], providing a persuasive argument for ensuring that optimal physical management is a clinical priority.

NICE Guidelines emphasise that mobility spans much more than simply walking [7], including activities necessary for daily functioning: the ability to stand, safely transfer from wheelchair to toilet or to bed, and to move in bed. People with MS who are severely impaired spend much of their day sitting, often with limited ability to change position. In response, secondary complications can occur rapidly, including muscle wasting, pain, reduced skin integrity, spasms, limb stiffness, constipation, and associated psychosocial problems such as depression and lowered self-esteem [8]. These secondary problems can accelerate the loss of independence in daily function, and can impact negatively upon quality of life $[4,9]$ and self-identity. The clinical significance of these issues is underlined by their consistent prominence in policy documents for long-term neurological conditions $[7,10]$.
There are also significant economic costs related to increasing immobility and secondary complications since they increase the burden of care, delay rehabilitation and increase healthcare costs. It is estimated that approximately $15 \%$ of people with MS will, at some point in time, develop a pressure sore [11], with treatment costs of a single pressure sore ranging from $£ 1,064$ to $£ 24,214$ [6]. The mean cost per wheelchair dependent patient is 4-5 times higher than an ambulatory patient [12]. This clearly has significant implications to the health services.

Evidence from studies of a range of neurological conditions, including MS, shows that increasing physical activity by interventions such as physiotherapy, can improve balance and mobility and reduce associated secondary health problems which arise from immobility [7]. However, increasing physical activity is difficult when disability is severe. Physiotherapy typically comprises short intermittent episodes of face-to-face sessions. Ongoing physiotherapy is rarely possible due to resource restrictions [13, 14]. While group-based exercise programmes are becoming more common, they can be inaccessible, and may have inappropriate content, for people with severe impairments [15]. Effective self-management strategies, which can be implemented relatively easily and cheaply within people's homes, are needed for severely impaired individuals to improve or maintain their ability to move and reduce their sedentary behaviour. However this is challenging, particularly in the latter stages of disease. Regular supported standing using standing frames is one potential self-management option.

A systematic review investigating effectiveness of supported standing in neurological conditions [8], provided some data to support its acceptability, feasibility and efficacy but highlighted that more robust evidence is required. Of 17 randomised controlled trials (RCTs), only two were of high quality, and only one trial involved people with MS [16], which was a short-term, hospital based programme. Our updated literature search additionally identified one mixed-methods study [17] and a qualitative study [18], but no further RCT's investigating this aspect of MS rehabilitation management. 
Among the work of this research team, a study be Hendrie et al., has provided compelling evidence of the need for a trial of a managed standing frame programme. This study used a mixed-methods approach involving nine in-depth single case studies [17] to provide an in-depth understanding of the experiences of people with MS who participated in the standing programme. Over the course of a year, in addition to a range of objective measures, Hendrie undertook 27 in-depth interviews. Respondents' stories reveal how the programme enabled them to "reconnect with their body", "regain lost skills", and gain a sense of "being in control". In line with other related research [18] these interviews provided convincing qualitative evidence that this self-management standing programme reinstated a sense of belonging and optimism about the future by restoring important life roles and feelings of normality. A particularly debilitating secondary complication of immobility is stiff/hypertonic muscles. Our laboratory-based studies [19] determined that the duration and magnitude of force required to ease the stiffness in hypertonic lower limb muscles could only be achieved in a supported standing position for more severely impaired people with MS

The proposed study builds on these existing research strands, asking the question "What is the clinical and cost effectiveness of a home-based self-management standing frame programme in people who are severely impaired with progressive MS?" The aim of this definitive multicentre randomised controlled study is to investigate the clinical and cost effectiveness of a home-based supported standing programme for people severely impaired with progressive MS.

More specifically, the objectives are to:

1. Assess the clinical effectiveness of the standing frame programme in improving motor function (primary outcome).

2. Assess the clinical effectiveness of the standing frame programme in improving balance, muscle strength, joint and muscle range, painful spasms, respiratory, bladder and bowel function, number of falls and quality of life (secondary outcomes).

3. Establish the cost-effectiveness of the standing frame programme versus usual care.

4. Explore the subjective experience of using a standing frame within the home, from the perspective of both the person with MS and their carer.

\section{Methods}

\section{Trial design}

This is a pragmatic multi-centre randomised controlled trial with blinded outcome assessment and full economic evaluation. Figure 1 shows the participant pathway.

\section{Study Setting}

Eight healthcare sites will be involved in this multicentred RCT which is based in two geographical regions of England: Devon/Cornwall and East Anglia. Each site consists of up to seven individual community based therapy teams.

\section{Participants \\ Sample size}

Our primary outcome is the Amended Motor Club Assessment (AMCA) at 16 weeks follow-up. Our primary analysis will utilise analysis of covariance (ANCOVA), comparing AMCA scores at week 36 between allocated groups, adjusting for baseline AMCA score. The trial is powered to detect a between-group difference of nine points which is both plausible and considered clinically relevant $[17,20]$. In people with severe MS a score change of 9 points is considered clinically significant, reflecting changes such as allowing a person to balance and lean forwards in sitting to dress themselves or to stand in the shower and wash their hair with two hands.

Estimates of final SD and baseline/follow-up correlation for AMCA are subject to uncertainty and thus we have used conservative $80 \%$ upper confidence limits for both parameters in our calculations. To detect a betweengroup difference of nine points, with $80 \%$ power and at the $5 \%$ significance level, requires data from 55 participants per group [21].

Allowance is required for people not completing the programme, and for participants not attending the final follow-up assessment. Based on our previous experiences, we have allowed for $20 \%$ loss to follow-up. Therefore the sample size required is 69 participants per group, rounded to a total of 140. An estimated 380 people will need to be screened to achieve the required sample size.

\section{Inclusion criteria}

The study population will comprise individuals diagnosed with primary or secondary progressive MS according to McDonald's criteria [22].

These participants will:

- be aged $>18$ years

- be willing and able to consent to participate

- score 6.5-8.0 on the Expanded Disability Status Scale (EDSS), i.e. people who "require bilateral assistance to walk 20 metres or less" to those "restricted to bed or wheelchair"

- be able to get into a standing frame independently or with assistance from one person

- have the agreement of another person (e.g. carer) should assistance be necessary for the standing programme 


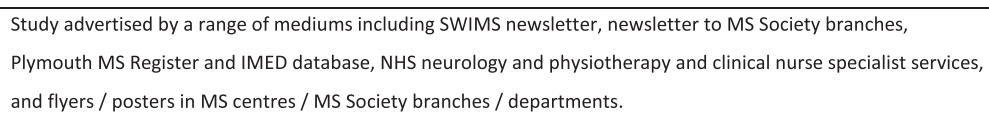

Telephone screens of the participant, their carer and local physiotherapist by the research therapist to ensure trial eligibility. Research therapist discusses the trial with the person with MS and their carer and answers any questions. Height details recorded for standing frame order. Appointment to attend a local health establishment arranged.

Participant attends and research therapist confirms eligibility, participant has an opportunity to ask further questions before providing signed consent, and undergoing baseline assessment.. Research therapist inputs participant data into web-based randomisation programme, and name of treating therapist to enable them to be notified of treatment group. All participants and their carers shown how to use the audio recorders in the event that they are allocated to the standing group audio diary sub-sample.

Automatic e-mail sent to treating therapist to notify them of participant allocation to Usual Care or Standing Intervention Group.

Control Group

Usual Care

Standing Intervention Group

Standing Frame ordered by the non-blinded research therapist in each region.

Treating therapist provides appointment to commence standing programme, within a maximum of two weeks following the blinded assessment.

Two face-to-face sessions (supplemented by a user friendly information booklet and DVD) undertaken by treating physiotherapist to set up the standing programme, as per protocol.

Trial administrator posts audio-recorders to the 10 carers and 10 participants who have agreed to complete the audio-diary.

Treating therapist provides telephone support to the participant on a weekly basis for the first month and on a monthly basis for the following two months (to monitor participant and carer, provide advice and optimise adherence by motivating the individuals to set and achieve personal targets related to the standing programme, and note adverse events).

\section{All participants}

Trial administrator arranges all participants' attendance at local health establishment to undergo the blinded assessments at two additional time-points ( 20 weeks post intervention and at follow-up 16 weeks later).

\section{All participants who discontinue use of the standing frame within the 36 week study period}

The regional study co-ordinators will telephone all participants and their carers who have decided not to continue with standing frame use over the course of the 36-week study period, to record reasons for stopping the programme.

Fig. 1 Trial flow chart of participant pathway 
- be able and willing to accommodate the standing frame in their home

- be willing and able to travel to local assessment centres for blinded outcomes assessment

\section{Exclusion criteria}

Participants will be excluded if they:

- have had any recent changes in disease modifying therapies. More specifically they will be excluded if: they have ever had Alemtuzumab, are within past six months of ceasing Nataluzimab, or are within three months of ceasing any other MS disease modifying drug

- have relapsed/received steroid treatment within the last month

- are currently undertaking a regular standing frame programme (>x1/week), or have done so during the past six months

- have a history of osteoporotic-related fractures

- have co-morbidities which contraindicate standing in the frame (e.g. foot ulceration, uncontrolled epilepsy) or likely to impact on the trial (e.g. chronic jaundice, heart disease, age related multiple co-morbidities)

- currently participating in another clinical trial (rehabilitation or pharmacological)

Identification and recruitment of participants will be via a number of routes which include screening MS databases, identification by healthcare professionals, and advertising via MS support groups and newsletters.

\section{Randomisation}

Participants will be randomised immediately after the baseline assessment. Randomisation, using random sized permuted blocks, will be on a 1:1 basis to receive the intervention plus usual care or usual care only. It will be stratified by region and baseline EDSS $(\leq 7.0$ or $\geq 7.5$ ). The allocation sequence is computer-generated by the local United Kingdom Clinical Research Collaboration Registered Peninsula Clinical Trials Unit (UKCRC PenCTU) (Registration Number 31) in conjunction with an independent statistician who will have no involvement with the final analysis. Following assessment, the blinded assessor will input the details of the participants directly into the randomisation web-site. This will generate an e-mail stating the participant's allocated treatment group to the regional study co-ordinators and study administrator to notify them of the treatment allocation.

\section{Blinding}

The trial participants and carers are unable to be blinded in this trial due to the nature of the intervention.
Similarly, the treating physiotherapists and health care providers are unable to be blinded. However, the outcome assessors will be blinded to participants' allocated group. All assessments will be undertaken in separate visits independently of delivery of treatment/usual care and away from the participant's home. Every effort will be made throughout the trial to ensure these assessments are blinded. Upon any interaction with the participant, the blinded assessors will remind participants not to discuss their allocated group, and this will be reiterated within any written and telephone correspondence.

At each assessment time point, the blinded assessor will be asked to record on a standardised form whether or not they were un-blinded to the group allocation, and if so the reasons for this.

\section{Interventions}

Participants in the intervention group will be asked to stand in an Oswestry standing frame [17] for $30 \mathrm{~min}$ three times per week for a total of 16 weeks during a 20 week period. The Oswestry standing frame is a wooden frame, used in clinical practice, which provides support in standing whilst allowing for exercise and controlled movement.

The treating physiotherapist will teach the participant and carer safe and effective use of the standing frame over two face-to-face sessions in the participant's home ( $60 \mathrm{~min} /$ session). Participants will also be taught exercises and stretches to undertake in the frame. A detailed user-friendly information leaflet and digital versatile disc (DVD) will be provided to support these sessions. Further support will be provided by weekly telephone contact for four weeks, and then monthly for two months. Telephone calls will focus on facilitating individuals to set and achieve personal targets.

\section{Standardisation and fidelity of the intervention}

Treating physiotherapists from each of the eight healthcare sites will perform the interventions as part of their NHS role; all physiotherapists will implement the intervention in a similar manner.

Use of standing frames is incorporated within undergraduate physiotherapy training and is a recognised core skill for neurological physiotherapists. To standardise and optimise implementation of the intervention, treating therapists, participants and carers will be provided with an information pack. This includes a written template of what is required to be undertaken within each session and a link to the study web-site, which houses a range of resources including a detailed booklet, instructional video with suggested exercise/stretches/balance activities, and evidence based articles on this topic. 
All treating therapists will be required to complete a pre-formatted checklist to self-assess their fidelity with the content of the sessions. They will be asked to record any deviations from the protocol on a Protocol Deviations form. In addition, $10 \%$ of the intervention sessions will be assessed in each region by the unblinded regional Principal Investigator who will observe the home-based treatment sessions and independently complete the same fidelity checklist.

\section{Usual care}

This trial will use a usual clinical care control group. A recent MS Society national survey demonstrated that access to physiotherapy services varies throughout the UK/ England [14]. Although usual care varies between individuals [1], it rarely involves regular physiotherapy intervention either within the community or hospital [13]. Any intervention is generally limited to a few visits, typically reacting to presenting problems (e.g. practising transfer skills, providing mobility aids) rather than promoting long-term preventative self-management [13]. This national picture reflects the care provided in our study regions. The usual care received (including frequency of physiotherapy intervention as well as any other health/social service interventions and medications) will be recorded within the economic cost effectiveness assessments.

\section{Data collection and outcome measurements}

Standardised, validated clinician-rated and patient selfreported clinical outcomes will be measured at baseline, immediately post intervention (20 weeks) and follow-up (week 36). Any deviations from this will be recorded on a Protocol Deviations form. The longer term follow-up is important to assess maintenance of any observed effect, and determine whether long-term engagement is sustained once support from the treating physiotherapist is withdrawn.

Measures have been selected on the basis of demonstrated reliability and validity in assessing physical impairments, clinical outcomes, quality of life, and economic costs in people with MS.

\section{Primary outcome measures}

The primary clinical outcome is motor function, assessed using the Amended Motor Club Assessment (AMCA) [23]. This rates motor impairment of the lower limb and trunk and key functional movements such as sit-to-stand and standing balance. It was developed specifically for people with MS and has demonstrated validity, reliability and responsiveness [20, 23, 24]

The primary economic endpoint is the quality-adjusted life year (QALY), assessed using the EuroQol five dimensional descriptive system, the EQ-5D-5 L self report measure [25].

\section{Secondary outcome measures}

A. Key clinical outcomes:

1. Bowel and bladder control using the self-report Bladder and Bowel Control Scales [26]

2. Sitting balance using the single item Modified Functional Reach in Sitting [27]

3. Falls frequency through a single yes/no question "Did you fall today?", recorded on the daily diary

4. Health-related quality-of-life (HRQoL), using the 29 item Multiple Sclerosis Impact Scale (MSIS-29 version 2.0) [28], a disease specific patient-reported outcome measure with a preference-based tariff [29] for use in sensitivity analyses for the Quality Adjusted Life Year (QALY) outcome.

B. Explanatory physical impairments:

1. Knee extensor strength using a portable hand-held dynamometer [30]

2. Length of hip flexors, hamstrings and ankle plantarflexors using manual goniometry [16]

3. Spasm frequency using the Penn Spasm Frequency Scale [31]

4. Respiratory capacity using a hand-held spirometer to record forced expiratory volume at one second [32]

\section{Measures of intervention adherence}

A simple pre-formatted daily diary will be completed by those allocated to the standing frame programme. Either the participant or carer will record adherence with the intervention (frequency, duration, reasons for not standing), and will also have the opportunity to comment on why standing was continued or stopped. Adverse events (including new symptoms and falls) will also be recorded in the diary.

The usual care group will also be asked to complete a daily diary to capture adverse events (including new symptoms and falls).

\section{Safety monitoring}

Participants will be monitored for adverse events via completion of their study daily diaries and during follow-up assessments. The group receiving the intervention will also be monitored during the scheduled telephone calls with their treating physiotherapist. Physiotherapists will be asked to report all adverse events to the research team, whether or not they are thought to be related to the intervention. 


\section{Economic evaluation}

The economic evaluation will estimate the cost effectiveness of the standing frame programme plus usual care, versus usual care alone. The primary perspective of the analyses will be that of the NHS and Personal Social Services (i.e. Third Party Payer), with a broader perspective to be considered in sensitivity analyses. Cost effectiveness analyses (CEA) will present an estimate of the incremental cost per unit change in the primary outcome measure (AMCA), but the primary economic analyses will be the incremental cost per QALY gained (over 36 week follow-up). The EQ-5D-5 L will be the primary economic endpoint used to estimate QALYs, applying a UK tariff [33], at final follow-up, with the MSIS-8D [29] used to estimate QALYS based on the MSIS-29, a condition specific measure. Economic analyses will estimate the resource use (e.g. standing frames, physiotherapy sessions, travel, telephone calls, social care assistance) and related cost associated with delivery of the standing frame programme, in addition to usual care, via data collected within the trial.

Data will be collected, via a self-report Resource Use Questionnaire, on participant use of health and social care services (primary, secondary and social care), and on broader aspects of participant and carer-related resource use.

Regression methods will be used to estimate incremental costs and QALYs, adjusting for baseline (cost, QALY) values, with covariates for baseline ACMA and stratification variables (baseline EDSS, geographic regions), and economic analyses will be consistent with the primary statistical analysis plan. The CEA will synthesise cost and outcome data, and explore uncertainty, to present results of the economic evaluation in a policy relevant way.

\section{Qualitative assessment}

A qualitative approach will be used to capture and explore the "real-life" experiences of people's participation in the standing frame programme. Purposive sampling to achieve maximum variation will be used to request 10 participants and 10 carers to keep audio diaries of their experiences of standing and using the frame. They will be asked to use an audio recorder to record their reflections and experiences on how it feels to stand, changes they are experiencing, plus any comments they wish to make throughout the intervention period. To ensure contemporaneous collection of data they will be asked to record these reflections, if possible, during each stand or as near to the completed standing period as possible.

\section{Statistical analysis}

A full statistical analysis plan will be developed and approved by an independent statistician, prior to final database-lock. The primary statistical analysis will utilise analysis of covariance (ANCOVA), comparing AMCA scores at week 36 between allocated groups, adjusting for baseline AMCA score and the two stratification variables (baseline EDSS category and geographical region). The primary comparative analyses of all outcome measures will be on the basis of intention-to-treat (adverse events will be presented per protocol if different). Between-group differences will be presented with $95 \%$ confidence intervals wherever possible, with the significance level for hypothesis testing set at $5 \%$, unless otherwise stated.

Secondary outcomes will be compared between groups in a similar way to that for the primary outcome. Comparisons of interest will be presented with $95 \%$ confidence intervals for both unadjusted and adjusted between-group comparisons.

\section{Subgroup analyses}

Exploratory analyses of the possible interaction between baseline EDSS and allocated group will be undertaken, to explore whether any difference between the allocated groups is modified by baseline EDSS category $(\leq 7.0$ vs $\geq 7.5$ ).

\section{Qualitative analysis}

Participants' stories will be gathered, analysed and reported using narrative methodology [34]. The texts from audio recordings will be left whole, crafted into a story, and not fractured into themes, as an attempt to capture the immediate impact of standing, and to form a chronological record of the person's experience. This promotes a contextual and holistic account; providing stories that are intended to resonate with people with MS, their families and professionals. By reading these stories the aim is to encourage the reader to reflect on their own situation gaining insight into their own experience as a person standing and living with MS, assisting a person to stand or as a professional.

\section{Data management, audit and monitoring}

Data will be recorded on study specific data collection forms by the research therapists. Completed forms will be entered onto a password-protected customised database, developed by the Peninsula CTU. All data will be double entered and compared for discrepancies. Discrepant data will be verified using the original paper data sheets.

Participants' anonymity will be maintained on all documents. Data will be collected and stored in accordance with the Data Protection Act 1998, and will be accessible for the purposes of monitoring, auditing, or at the request of the regulatory agency. 


\section{Trial management committees}

There are two trial management groups involved in the set up and management of this trial: the Trial Management Group (TMG) and the Trial Steering Committee (TCS).

The TMG comprises 10 individuals involved in the study design and protocol development. The group will meet approximately monthly to oversee the general management of the day to day running of the trial and release the trial results and publications. Closely involved with the sponsors, most members are coinvestigators on this study. In contrast the decisions made by the TSC are independent of the sponsors and investigators. The TSC, which meets annually, comprises eight individuals, with majority independent representation (chair, external statistician, member of MS Society and two independent lay members. In addition the chair of the TSC will receive a quarterly update of the adverse events, and a telephone conference / additional face-toface meeting will be instigated by the Chair or the Chief Investigator should any issues need to be discussed.

The role of the TSC is to oversee the conduct of the trial, including for instance monitoring adverse events, recruitment and attrition rates, the project timeline and finances.

\section{Ethics}

The trial will be conducted in accordance with the ethical principles that have their origin in the Declaration of Helsinki, 1996; the principles of Good Clinical Practice, and the Department of Health Research Governance Framework for Health and Social Care, 2005. The study protocol, participant information and enrolment procedures were assessed and approved through the National Research Ethics Scheme (NRES Committee South West Frenchay, REC ref no. 15/SW/0088) and management permissions gained from the Research and Development Departments of the eight participating centres in accordance with NHS research governance arrangements. Any amendments to the protocol will be reported to, and approved via NRES.

\section{Dissemination plan}

We will target users, clinicians, researchers, organisations developing clinical guidelines and NHS decision makers. On completion of the trial, the full study report will be accessible on the study web-site page, as will the full protocol. This protocol (Version 3.0, dated 5.2.2015) has been published in line with SPRIT Guidelines [35]. Similarly CONSORT (Consolidated Standards of Reporting Trials) [36] and The Template for Intervention Description and Replication (TiDIER) Guidelines [37] will be reviewed prior to submitting future publications of the trial results to high quality journals. Authorship of intended articles will be by the study team; professional writers will not be used. Results will be presented at national and international conferences to ensure dissemination to academics and those responsible for service delivery. In addition results will be disseminated through the newsletters of MS organisations and via talks to their local support groups. If proven effective, the training materials (treatment manual, instructional video recordings, case scenarios) will be made freely available via the study website, with the aim of optimising roll out. Extracts from the audio recordings will also be used as educational and decision making tools for use by both people with MS and therapists involved in their care. All participants, who consent to receiving notifications, will be notified in writing of trial outcomes.

\section{Discussion}

Preliminary evidence from two small-scale studies suggests that the use of standing frames in the home may improve physical and psychological well-being in people with MS $[16,17]$. This protocol describes the first largescale trial to evaluate the use of the standing frame in this way. It is important to determine definitively whether such a programme is clinically and cost effective so that people affected by MS, clinicians, commissioners and policy makers can make informed evidence-based decisions regarding the use of this intervention.

Some of the choices in the study design warrant discussion. First we discuss why our inclusion criteria were confined to those individuals with progressive disease. This is for two main reasons: the lack of diseasemodifying drugs for progressive MS which means that management is aimed solely at minimising symptoms and, if possible, improving function; and the paucity of studies devoted to improving mobility in people with progressive MS [38]. As a consequence there are few proven interventions for these people. By testing the use of standing frames in a multi-centre randomised controlled trial, we may be able to identify a successful and cost effective intervention. In doing so, the results have the potential to change clinical practice and optimise supported self-management within a population that receive little attention, yet are severely affected by their condition.

The second relevant issue is the stratification by region and baseline disability level (as determined by the EDSS $\leq 7.0$ or $\geq 7.5$ ). We stratified by region (South West England versus East Anglia) because of possible differences in terms of usual care provided. Stratification by disability level was included because of the potential differences in ability of participants to adhere to the intervention, and respond to it. We hypothesise that those with higher levels of disability will have lower levels of adherence and be at higher risk of drop out of the study. 
Furthermore, more severely impaired participants may utilise greater levels of health and social service provision which may bias the cost effectiveness and cost-utility analyses.

Third, the dose (frequency and intensity) of the selfmanagement standing intervention and the time frame over which this was increased was informed by a combination of scientific and anecdotal evidence. The standing duration of 20 weeks factors in up to four weeks for individuals to become gradually re-accustomed to an upright position and to steadily achieve the desired intensity of standing (three times a week for $30 \mathrm{~min}$ per session over 16 weeks) which was based on previous studies $[16,17]$. In doing so, we hope that it will minimise fatigue, and allow for time when the participant is unable to use the frame (illness, holidays, etc.), as highlighted by our user discussion groups.

Fourth, a practical issue that we considered was which of the various commercially available standing frames we would utilise for this study. The Oswestry Standing Frame was chosen because: (a) it is commonly used within both the National Health Service, the charitable sector (e.g. MS Centres), and within undergraduate physiotherapy teaching, hence minimising the training requirements for clinicians; (b) it is both durable and recyclable, and hence can be recirculated for use within the health service thereby enhancing sustainability; (c) it is relatively lightweight (the frame can easily be pushed across a floor by one person) which makes it easy to move into a position for standing in the home and then returned to a storage part of the room when not in use; (d) it is used within current clinical practice in a range of conditions (e.g. stroke, spinal cord injury, cerebral palsy) and hence, should the results of this trial be negative, then it could continue to be recycled for use within the health service; (e) it is one of the cheapest frames on the market, and finally (e) it is wooden and hence perceived by some patients as "more like a piece of furniture", which is an important consideration given that these frames will be used within people's homes.

\section{Trial status}

Recruitment started in September 2015 and is ongoing (49 participants recruited as of 21st March 2016).

\section{Ethics approval and consent to participate}

The study protocol, participant information and enrolment procedures were assessed and approved through the National Research Ethics Scheme (NRES Committee South West - Frenchay, REC ref no. 15/SW/0088) and management permissions gained from the $\mathrm{Re}$ search and Development Departments of the eight participating centres in accordance with NHS research governance arrangements. Any amendments to the protocol will be reported to, and approved via NRES.

\section{Availability of data and material}

Data generated as a result of this study, and on which the conclusions of future manuscripts will be relied, will be presented in the main paper or additional supporting files.

\section{Abbreviations}

AMCA: Amended Motor Club Assessment; ANCOVA: analysis of covariance; CEA: cost effectiveness analyses; CONSORT: Consolidated Standards of Reporting Trials; CTU: Clinical Trials Unit; DVD: digital versatile disc; SUMS: Standing Up in Multiple Sclerosis; EDSS: Expanded Disability Status Scale; HRQoL: Health-related quality-of-life; MSIS: Multiple Sclerosis Impact Scale; NHS: national health service; NRES: National Research Ethics Scheme; QALY: quality adjusted life year; RCTs: randomised controlled trials;

SUMS: multiple sclerosis; TCS: trial steering committee; TiDIER: Template for Intervention Description and Replication; TMG: trial management group; UK: United Kingdom; UKCRC: United Kingdom clinical research collaboration.

\section{Competing interests}

The authors declare that they have no competing interests.

\section{Authors' contributions}

$J F, W H, L J, A B, S C, C G, J M, J Z$ designed the overall study. SC provided the statistical power calculation and sample size calculation, JF, WH, ER, LJ, AB, SC, CG wrote the manuscript. JF, WH, LJ, SC wrote the study protocol. JM and JZ critically proof-read the manuscript and provided input to the study design. All authors read and approved the final manuscript.

\section{Funding and sponsorships}

This study is funded by the NIHR Research for Patient Benefit Programme (PB-PG-1013-32047), United Kingdom. This article presents independent research. The views expressed are those of the authors and not necessarily those of the NHS, the NIHR or the Department of Health. Sponsor of this clinical study is the Plymouth Hospitals NHS Trust, Devon, England, UK.

\section{Author details}

${ }^{1}$ Faculty of Health and Human Sciences, School of Health Professions, Plymouth University, Peninsula Allied Health Centre, Derriford Rd, Plymouth PL6 8BH, England. ${ }^{2}$ Norwich MS Centre, Alkmaar Way, Norwich NR6 6BB, England. ${ }^{3}$ Centre for Health Statistics, Peninsula Schools of Medicine and Dentistry, Room 302, Tamar Science Park, Plymouth PL68BX, England. ${ }^{4}$ Mardon Neurorehabilitation Centre, Royal Devon and Exeter NHS Foundation Trust, Wonford Rd, Exeter EX2 4UD, England. ${ }^{5}$ Research and Design Service, Peninsula Schools of Medicine and Dentistry, ITTC Building, Tamar Science Park, Plymouth PL68BX, England. ${ }^{6}$ University of Exeter Medical School, Health Economics Group, University of Exeter, Veysey Building, Exeter, Devon EX24SG, England. 'School of Medicine, Medical and Biological Sciences, University of St Andrews, North Haugh, St Andrews, Scotland KY169TF, UK.

Received: 1 April 2016 Accepted: 28 April 2016

Published online: 05 May 2016

\section{References}

1. Royal College of Physicians The national audit of services for people with multiple sclerosis 2011. Royal College of Physicians, London. Available at https://www.rcplondon.ac.uk/projects/national-audit-services-peoplemultiple-sclerosis (Accessed April 2016)

2. McCrone $P$, Heslin $M$, Knapp $M$, et al. Multiple sclerosis in the UK: service use, costs, quality of life and disability. Pharmaco Econ. 2008;26:847-60.

3. Giesser BSE. Primer on Multiple Sclerosis. 2011. Oxford University Press.

4. Jones SL, Warren S, Turpin KVL, et al. The burden of multiple sclerosis: A community health survey. Health Quality Life Outcomes. 2008;6:1. doi:10.1186/1477-7525-6-1.

5. Sutliff $\mathrm{MH}$. Contribution of impaired mobility to patient burden in multiple sclerosis. Current Med Res Opinion. 2010;26:109. 
6. Heesen CBJ, Reich C, Kasper J, et al. Patient perception of bodily functions in multiple sclerosis: Gait and visual function are the most valuable. Mult Scler. 2008;14:998-1.

7. National Institute of Clinical Excellence. Multiple Sclerosis: management of multiple sclerosis in primary and secondary care. NICE Clinical Guideline 186, October 2014. Available at: http://www.nice.org.uk/guidance/cg186 (Accessed April 2016)

8. Newman M, Barker K. The effect of supported standing in adults with upper motor neurone disorders: a systematic review. Clin Rehabil. 2012;26(12):1059-77.

9. Irvine H, Davidson C, Hoy K, Lowe-Strong A. Psychosocial adjustment to multiple sclerosis: exploration of identity redefinition. Disabil Rehabil. 2009;31(8):599-606.

10. Department of Health. The National Service Framework for Long Term Conditions. 2005 Department of Health, London. Accessible at https://www. gov.uk/government/uploads/system/uploads/attachment_data/file/198114/ National_Service_Framework_for_Long_Term_Conditions.pdf (Accessed April 2016)

11. Cramp AFL, Warke K, Lowe-Strong AS. The incidence of pressure ulcers in people with multiple sclerosis and persons responsible for their management. Int J MS Care. 2004;6(2):52-4.

12. Kobelt $G$, Berg J, Lindgren $P$, et al. Costs and quality of life of multiple sclerosis in the United Kingdom. Eur J Health Econ. 2006;7:S96-104.

13. Forbes A, While A, Taylor M. What people with multiple sclerosis perceive to be important to meeting their needs. J Adv Nurs. 2007;58(1):11-22.

14. MS Society of Great Britain. A lottery of treatment and care: MS services across the UK. April 2013. Accessible at: http://mslottery.mssociety.org.uk/ wp-content/uploads/2013/04/UK-ms-lottery.pdf (Accessed April 2016).

15. Hale LA, Smith C, Mulligan H, et al. "Tell me what you want, what you really really want"... asking people with multiple sclerosis about enhancing their participation in physical activity. Disabil Rehabil. 2012;34(22):1887-93.

16. Baker K, Cassidy E, Rone-Adams S. Therapeutic standing for people with multiple sclerosis: Efficacy and feasibility. Int J Ther Rehabil. 2007;14(3):104-9.

17. Hendrie WA, Watson MJ, McArthur MS. A pilot mixed methods investigation of the use of Oswestry standing frames in the homes of nine people with severe multiple sclerosis. Disabil Rehabil. 2014;37(13):1178-85.

18. Nordstrom B, Naslund A, Eriksson A, et al. The impact of supported standing on wellbeing and quality of life. Physiother Can. 2013a;65(4):49-57.

19. Marsden J. Effect of stretching on stiffness and range of motion in people with multiple sclerosis (Abstract). London: MS Frontiers Conference; 2013. Available at http://docslide.us/health-medicine/ms-frontiers-2013-brochure. html (Accessed April 2016).

20. Craig J, Young C, Ennis M, et al. A randomised controlled trial comparing rehabilitation against standard therapy in multiple sclerosis patients receiving intravenous steroid treatment. J Neurol Neurosurg Psychiatry. 2003;74(9):1225-12.

21. Borm GF, Fransen J, Lemmens WAJG. A simple sample size formula for analysis of covariance in randomized clinical trials. J Clin Epidemiol. 2007;60:1234-8.

22. Polman $\mathrm{CH}$, Reingold SC, Banwell B, et al. Diagnostic criteria for multiple sclerosis:2010 Revisions to the McDonald criteria. Ann Neurol. 2011;69:292-302.

23. De Souza LH, Ashburn A. Assessment of motor function in people with multiple sclerosis. Physiother Res Int. 1996;1(2):98-111.

24. Davis C. Physiotherapy or Self-Selected Exercise in Multiple Sclerosis, A Comparative Evaluation of Community-based Interventions. PhD Thesis, University of Nottingham 2008. Accessible at: http://eprints.nottingham.ac. uk/12379/ (Accessed April 2016)

25. Herdman M, Gudex C, Lloyd A, et al. Development and preliminary testing of the new five-level version of the EQ-5D (EQ-5D-5 L). Qual Life Res. 2011;20:1727-36.

26. National MS Society. The Consortium of Multiple Sclerosis Centers Health Services Research Subcommittee. Multiple sclerosis quality of life inventory: a user's manual. Bowel Control Scale (BWCS) 1997. Available at http://walkcoc.nationalmssociety.org/docs/HOM/MSQLI_Manual_and_ Forms.pdf. (Accessed April 2016)

27. Katz-Leurer MI. Fisher et al. Reliability and validity of the modified functional reach test at the sub-acute stage post-stroke. Disabil Rehabil. 2009;31(3):243-8.
28. Hobart J, Lamping D, Fitzpatrick R, et al. The Multiple Sclerosis Impact Scale (MSIS- 29): A new patient-based outcome measure. Brain. 2001;124(5):962-73.

29. Goodwin E, Green C, Spencer A. Estimating a preference-based index for an eight dimensional health state classification system derived from the Multiple Sclerosis Impact Scale (MSIS-29). Value Health. 2015;18:1025-36.

30. Surakka JF, Romberg A, et al. Assessment of muscle strength and motor fatigue with a knee dynamometer in subjects with multiple sclerosis: a new fatigue index. Clin Rehabil. 2004;18(6):652-9.

31. Guillaume D, Van Havenbergh A, Vloeberghs M, et al. A clinical study of intrathecal baclofen using a programmable pump for intractable spasticity. Arch Phys Med Rehabil. 2005;86(11):2165-71.

32. Buyyse B, Demedts M, Meekers J, et al. Respiratory dysfunction in multiple sclerosis: a prospective analysis of 60 patients. Eur Respir J. 1997;10:139-45.

33. Mulhern B, Bansback N, Brazier J, et al. Preparatory study for the re-valuation of the EQ-5D tariff: Project report. Health Technol Assess. 2014;18(12):vii-Xxvi. ISSN 1366-5278.

34. Clandinin DJ, Connelly FM. Narrative inquiry. Experience and story in qualitative research. San Francisco: Jossey-Bass; 2000

35. Chan A-W, Tetzlaff JM, Gøtzsche PC, Altman DG, Mann H, Berlin J, Dickersin K, Hróbjartsson A, Schulz KF, Parulekar WR, Krleža-Jerić K, Laupacis A, Moher D. SPIRIT 2013 Explanation and Elaboration: Guidance for protocols of clinical trials. BMJ. 2013;346:e7586

36. Moher D, Hopewell S, Schulz KF, Montori V, Gøtzsche PC, Devereaux PJ, Elbourne D, Egger M, Altman DG, for the CONSORT Group. CONSORT 2010 Explanation and Elaboration: updated guidelines for reporting parallel group randomised trial. BMJ. 2010;340:c869.

37. Hoffmann T, Glasziou P, Boutron I, Milne R, Perera R, Moher D, Altman D, Barbour V, Macdonald H, Johnston M, Lamb S, Dixon-Woods M, McCulloch P, Wyatt J, Chan A, Michie S. Better reporting of interventions: template for intervention description and replication (TIDieR) checklist and guide. BMJ. 2014;348:g1687. doi:10.1136/bmj.g1687.

38. Feinstein A, Freeman J, Lo AC. Treatment of progressive multiple sclerosis: what works, what does not, and what is needed. Lancet Neurol. 2015;14(2):194-207.

\section{Submit your next manuscript to BioMed Central and we will help you at every step:}

- We accept pre-submission inquiries

- Our selector tool helps you to find the most relevant journal

- We provide round the clock customer support

- Convenient online submission

- Thorough peer review

- Inclusion in PubMed and all major indexing services

- Maximum visibility for your research

Submit your manuscript at www.biomedcentral.com/submit
) Biomed Central 